\title{
Note
}

\section{Effets de l'adjonction de lipides dans la ration sur les performances de ponte et l'efficacité alimentaire de poules normales ou naines}

\author{
A. EL-ATTAR ${ }^{(*)}$, A. BORDAS, P. MÉRAT \\ Laboratoire de Génétique Factorielle, I.N.R.A. \\ Centre National de Recherches Zootechniques, \\ F 78350 Jouy-en-Josas
}

\begin{abstract}
Résumé
Les performances de ponte et d'efficacité alimentaire ont été comparées pour des poules Leghorn de taille normale $\left(D w^{+}\right)$ou naines $(d w)$ en présence de 2 régimes à teneurs différentes en lipides totaux. Le régime le plus riche a, dans l'ensemble, un effet négatif sur la variation de poids corporel des pondeuses, du moins pour les poules de taille normale. Pour les poules naines, d'autre part, l'intensité de ponte semble comparativement un peu plus améliorée par le régime enrichi en lipides que pour les poules de génotype $D w^{+}$. Parallèlement, les effets des mêmes régimes alimentaires ont été comparés pour des poules toutes naines appartenant à une lignée de type " œuf brun ". Au total, il n'apparaît pas d'avantage décisif du régime enrichi en lipides, et les interactions génotype $\times$ régime sont d'importance limitée.
\end{abstract}

Mots clés: Poule, nanisme lié au sexe, ponte, lipides.

\section{Summary}

Effects of the addition of lipids in the feed on laying performances and feed efficiency of normal and dwarf hens

Egg production traits and feed efficiency were compared for normal-sized $\left(D w^{+}\right)$or dwarf (dw) White Leghorn hens in 2 groups receiving diets with different levels of total lipids. The diet with higher lipid content had on the whole a negative effect on body weight variation of layers, at least for the normal-sized hens. On the other hand, laying intensity of the dwarf hens seemed to be slightly more improved by the addition of fat in the diet for dwarfs than for hens of the $D w^{+}$ genotype. At the same time, the same 2 feeds were compared on dwarf females belonging to a brown-egg type strain. On the whole, the diet with higher lipid content has no decisive advantage for either genotype, and the genotype $\mathrm{x}$ diet interactions are of limited importance.

Key words : Hen, sex-linked dwarfism, egg production, lipids.

$\left(^{*}\right)$ Adresse permanente : Département de Production Animale, Faculté d'Agriculture, Université AïnShams, Le Caire, Egypte. 


\section{Introduction}

Dans un précédent travail (DEmarne et al., 1984), une différence entre des poules naines $(d w)$ et normales $\left(D w^{+}\right)$avait été mise en évidence pour la composition en acides gras des triglycérides du jaune de l'œuf. Ce dernier était relativement plus riche en acide linoléique (provenant totalement de l'aliment) et moins riche en acide oléique chez les poules naines que chez leurs sœurs normales. Nous avons, pour notre part, voulu comparer les conséquences de l'adjonction d'un supplément de lipides totaux et en particulier d'acide oléique dans la ration sur les performances de ponte et l'efficacité alimentaire de poules de ces 2 génotypes. Ses conséquences sur les divers aspects du métabolisme des lipides seront décrites par ailleurs.

\section{Matériel et méthodes}

\section{A. Animaux - Schéma expérimental}

Les poules étaient issues d'une population Leghorn blanche dans laquelle le gène de nanisme lié au sexe $d w$ était maintenu en ségrégation par l'accouplement de coqs hétérozygotes $D w^{+} d w$ avec des poules $d w$. Parmi les filles de chaque génotype, écloses en septembre 1983 et mises en cages individuelles à l'âge de 18 semaines, la moitié recevait à partir de cet âge une ration "pondeuses » avec adjonction de lipides supplémentaires, l'autre moitié recevait un régime témoin peu riche en lipides. La composition des 2 régimes figure au tableau 1.

\section{Tableau 1}

Composition des régimes.

Composition of feeds.

\begin{tabular}{|c|c|c|}
\hline Composant (p. 100) & Régime témoin & $\begin{array}{l}\text { Régime enrichi } \\
\text { en lipides }\end{array}$ \\
\hline 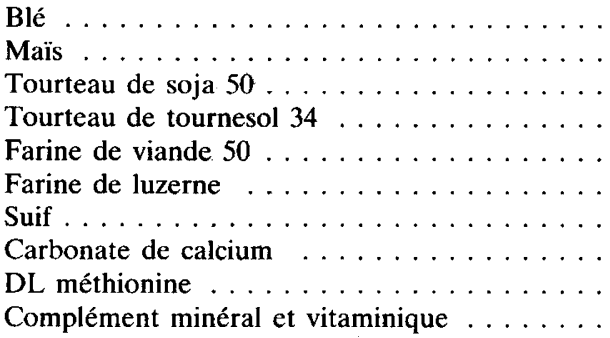 & $\begin{array}{r}21,5 \\
45,0 \\
6,0 \\
6,0 \\
5,0 \\
6,0 \\
- \\
8,0 \\
0,1 \\
2,5\end{array}$ & $\begin{array}{r}16,2 \\
45,0 \\
8,0 \\
11,3 \\
5,0 \\
- \\
4,0 \\
8,0 \\
0,1 \\
2,5\end{array}$ \\
\hline 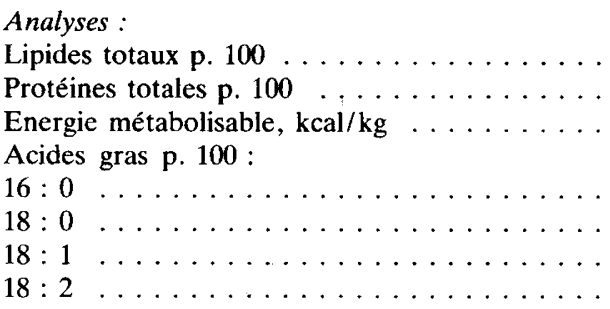 & $\begin{array}{r}1,9 \\
15,5 \\
2520 \\
\\
0,25 \\
0,06 \\
0,47 \\
0,98\end{array}$ & $\begin{array}{r}4,2 \\
15,9 \\
2770 \\
\\
0,89 \\
0,49 \\
1,37 \\
1,11\end{array}$ \\
\hline
\end{tabular}


Parallèlement, une population de type « œuf coloré » fixée pour le gène dw a été répartie en cages individuelles dans le même local, 97 et 96 de ces poules recevant respectivement l'aliment enrichi en lipides et l'aliment témoin.

\section{B. Méthodes statistiques}

Pour les poules Leghorn, une analyse de variance à 2 facteurs permet de tester les effets « régime " et " génotype " et leur interaction. Dans la population à œufs teintés, seul l'effet " régime » (analyse de variance à un facteur) est testé.

\section{Résultats et discussion}

Le tableau 2 indique pour la population Leghorn les valeurs moyennes par génotype et par régime pour des caractéristiques de ponte et d'efficacité alimentaire et les tests de signification des effets génotype et régime. Parmi les variables associées à l'efficacité alimentaire, la consommation alimentaire "résiduelle » représente l'écart de la consommation observée à l'estimation tirée d'une équation de régression multiple sur le poids corporel, sa variation et la masse d'œufs. Les performances des poules naines de type « œuf coloré » relatives à chaque régime sont données également à titre indicatif.

Le présent essai visait essentiellement à comparer la réponse de poules naines et normales à une différence du taux de lipides dans la ration, car nous ue connaissons pas de résultats publiés sur ce point. Nous ne commenterons donc que brièvement les effets associés au gène $d w$ indépendamment du régime. Ces effets, hautement significatifs quant à la réduction du poids corporel, du poids moyen des œufs, de l'intensité de ponte, de la longueur des séries, de la masse des oufs, de la consommation d'aliment, enfin du pourcentage d'œufs anormaux, sont bien connus dans les souches de type « ponte ». En particulier, la réduction de la masse d'œufs est ici très forte (plus de 40 p. 100) : ceci avait déjà été signalé (BANERJEe et al., 1981) et mis en rapport avec la taille particulièrement petite de la lignée Leghorn utilisée.

Quant aux effets attribuables au régime dans la lignée Leghorn, la diminution significative de l'indice de consommation et celle de la variation de poids du génotype $D w^{+}$avec la ration enrichie en lipides peut provenir, outre cet apport spécifique, du fait que cette ration est plus énergétique que la ration témoin. Dans la population naine type « œufs teintés », l'effet du régime enrichi est de même sens pour le gain de poids et l'indice de consommation; en outre, la consommation "résiduelle » est réduite. D'autre part, l'effet des lipides du régime sur le poids de l'œuf a été discuté (p. ex. LeClerce, 1973). Dans le cas présent, la teneur en lipides totaux de la ration n'influe pas significativement sur ce critère, quoique dans les 2 populations, le poids moyen de l'œuf des naines paraisse accru par l'apport de lipides supplémentaires.

En ce qui concerne la réponse comparée des poules Leghorn $D w^{+}$et $\mathrm{dw}$ au régime, seules 2 variables montrent une interaction génotype $\times$ régime significative au seuil 5 p. 100 : la variation de poids en 28 jours et l'intensité de ponte. Chez les poules de taille normale seules, la variation de poids est nettement diminuée par le régime 


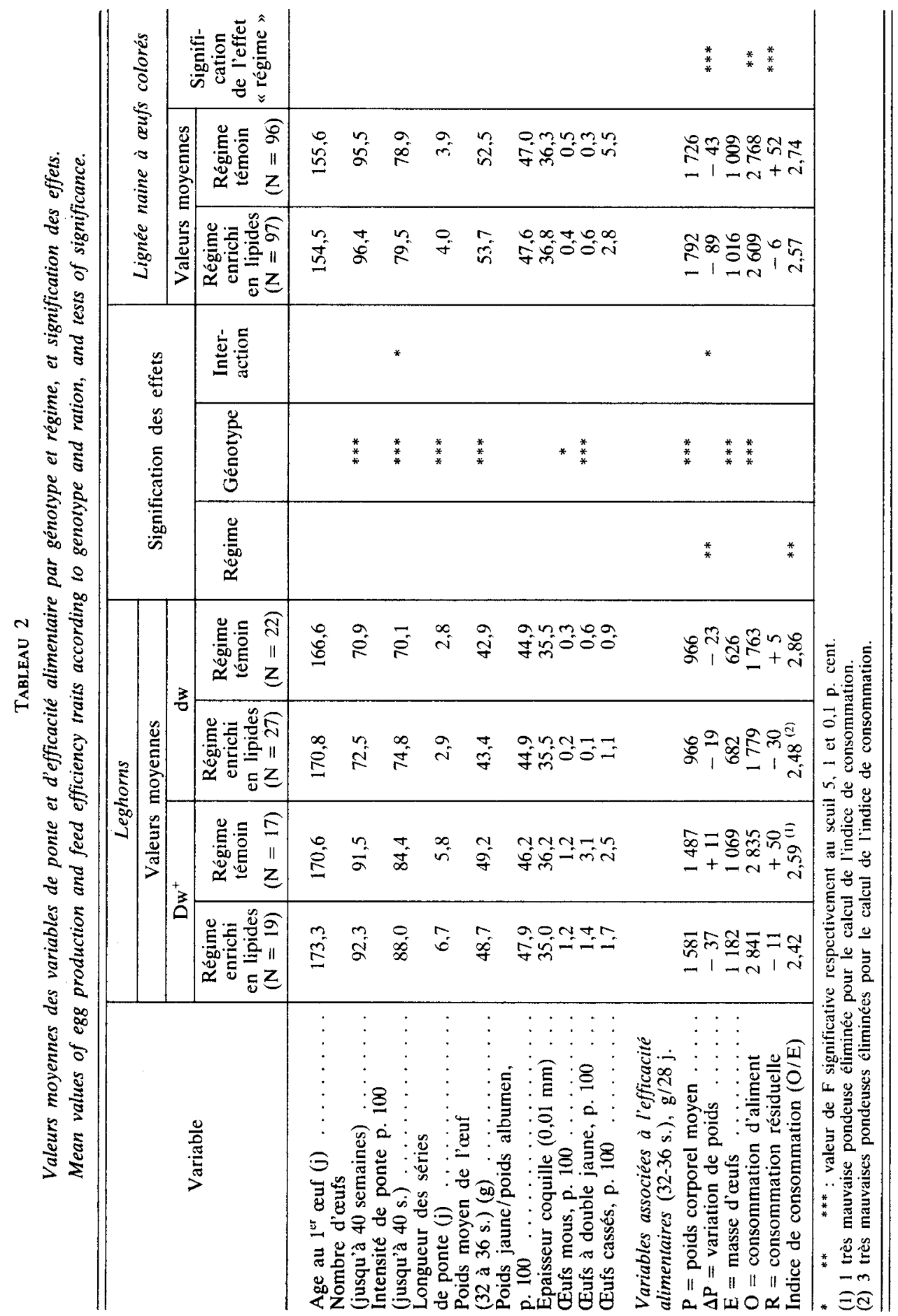


plus riche en lipides ${ }^{(1)}$. L'intensité de ponte paraît légèrement améliorée par ce même régime dans les 2 génotypes, mais un peu plus chez les poules $d w$. Les tendances semblent de même sens dans la lignée de type " œuf coloré », quoique faiblement en ce qui concerne l'intensité de ponte. Par contre, on ne trouve pas d'interaction pour le nombre et la masse d'œufs et l'indice de consommation. Quoique les valeurs du tableau 2 suggèrent que les poules $d w$ bénéficient un peu plus du régime enrichi que les poules $D w^{+}$pour ce dernier critère, cela ne se traduit pas par une interaction significative. A cet égard, les naines ne paraissent pas bénéficier davantage d'un apport de lipides totaux que les poules normales. Rappelons que les lipides plasmatiques totaux ne diffèrent pas entre ces génotypes (DEmarne et al., 1984). Si l'on tient d'ailleurs compte de la différence d'énergie métabolisable des 2 aliments et remplace l'indice de consommation par le rapport de l'énergie consommée (kcal) à la masse d'œufs produite $(\mathrm{g})$, ce rapport est le suivant : respectivement 6,66 et 6,68 pour les poules $D w^{+}$avec l'aliment enrichi en lipides et l'aliment témoin, et 7,22 et 7,10 pour les poules $d w$ avec ces mêmes aliments. Pour cet « indice de consommation énergétique ", les poules $d w$ apparaissent inférieures aux pondeuses de taille normale, mais l'aliment paraît n'avoir aucun effet et il n'apparaît aucune interaction entre génotype et aliment. Peut-être faudrait-il tenir compte de l'apport respectif de certains acides gras dans chaque régime : le plus riche contient relativement moins d'acide linoléique $(26,5$ contre 51,7 p. 100$)$ et davantage d'acide oléique $(32,7$ contre 25,5 p. 100$)$ que l'autre ; en valeur absolue, il apporte un supplément important du second. Sachant par ailleurs la différence entre poules $D w^{+}$et $d w$ pour le dépôt de ces acides gras dans l'œuf (DEMARNe et al., 1984), des recherches ultérieures devraient comparer ces génotypes selon l'apport respectif de ces acides gras dans la ration.

Reçu le 16 septembre 1985.

Accepté le 18 avril 1986.

\section{Références bibliographiques}

Banerjee A.K., Bordas A., Mérat P., 1981. Sex-linked dwarf gene $(d w)$ in White Leghorn laying hens under normal or hot temperature. Ann. Génét. Sél. Anim., 14, 135-160.

Demarne Y., Mérat P., Pinet A., 1984. Composition des lipides de l'œuf chez des poules Leghorn normales et naines. Génét. Sél. Evol., 16, 211-220.

Leclerco B., 1973. Contribution de l'aliment et des réserves corporelles à la genèse des lipides vitellins de la poule. Thèse Doctorat ès Sciences, Université Paris V.

(1) F entre régimes chez les poules $D w^{+}=13,3(\mathrm{p}<0,001)$; NS chez les poules $d w(\mathrm{~F}=1,1)$. 